\title{
ARBOLES DE SOMBRA E INTENSIDAD DEL CULTIVO AFECTAN EL RENDIMIENTO DE CAFÉ (Coffea arabica L.) Y LA VALORACIÓN ECOLÓGICA EN MASATEPE, NICARAGUA
}

\author{
SHADE TREES AND INTENSITY OF MANAGEMENT AFFECT YIELD OF COFFEE \\ (Coffea arabica L.) AND THE ECOLOGICAL ASSESSMENT IN MASATEPE, NICARAGUA
}

\begin{abstract}
Moraga Quezada Pedro1, Bolaños Taleno Roger llich", Pilz Mario ${ }^{3}$, Munguía Hernández Rodolfo4, Jürgen Pohlan Hermann Alfred ${ }^{5}$, Barios Mirna ${ }^{6}$, Haggar Jeremy ${ }^{6}$, Gamboa Moya William ${ }^{7}$

${ }^{1}$ Centro de Capacitación y Servicios Regionales del Café del Pacífico Sur / UNICAFE, Nicaragua. ${ }^{2}$ Instituto Nicaragüense de Tecnología Agropecuaria / INTA, Nicaragua. ${ }^{3}$ Estudiante en la Universidad Bonn, Alemania. ${ }^{4}$ Universidad Nacional Agraria / UNA, Nicaragua. ${ }^{5}$ Consultor Independiente, Alemania. ${ }^{6}$ Centro Agronómico Tropical de Investigación y Educación / CATIE, Nicaragua. ${ }^{7}$ Consultor Independiente, Costa Rica.
\end{abstract}

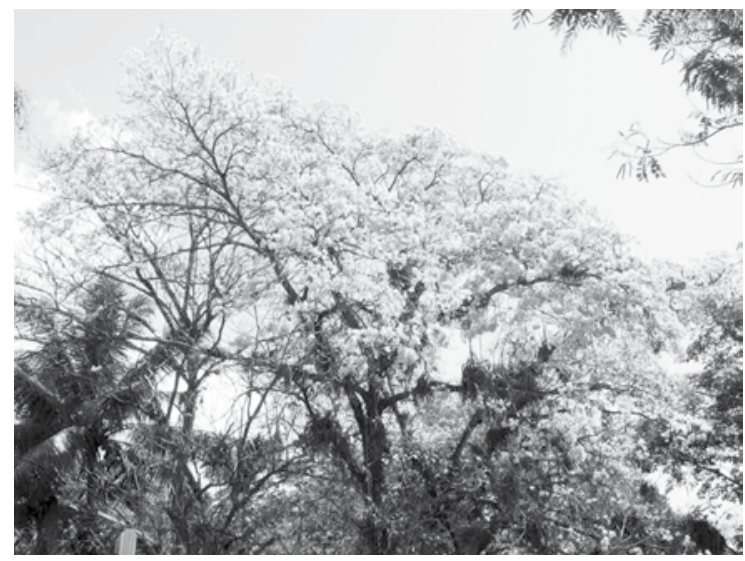

\section{RESUMEN}

En el año 2000 se establecieron dos repeticiones de un ensayo de café, en el Centro de Capacitación y Servicios Regionales del Café del Pacífico Sur - UNICAFE; una tercera repetición fue establecida en el año 2001 en el Centro Experimental Campos Azules (CECA) del INTA, en el Municipio de Masatepe, departamento de Masaya, Nicaragua. El propósito general del ensayo fue evaluar la influencia de diferentes tipos de sombra: Simaruba glauca + Tabebuia rosea (SGTR), Simaruba glauca + Inga laurina (SGIL), Samanea Saman + Tabebuia Rosea (SSTR), Inga laurina + Samanea saman (ILSG) y un cafetal a Pleno sol (PS), con dos niveles de insumo Convencional intensivo (CI) y Convencional moderado (CM), sobre la producción y rendimiento de café oro y la valoraron de los servicios ambientales como Biodiversidad, Captura de carbono, Conservación de suelo y de agua. Además, se incluyeron los tratamientos orgánico intensivo (OI) y Orgánico moderado $(\mathrm{OM})$. Se determinó que la sombra afectó la producción de café oro, pero mejoró el rendimiento en comparación al cultivo a pleno sol. La combinación de sombra de SGTR interactuando con las aplicaciones de insumos orgánicos intensivos, registró mejor producción promedio $(2674 \mathrm{~kg}$ oro ha $^{-1}$ ) de la cosecha 5, superando al tratamiento a pleno sol con uso de insumos convencionales intensivo. Cafetales bajo

\section{ABSTRACT}

The study was carried out in the community of Masatepe, department of Masaya, Nicaragua where in 2000 a longterm coffee experiment was established with two repetitions in the Centro de Capacitación y Servicios Regionales del Café del Pacífico Sur (Jardín Botánico) part of the Unión Nicaragüense de Cafetaleros (UNICAFE) and a third repetition in the Centro Experimental de Campos Azules (CECA) of the INTA. The purpose of the research was to study the production, yield and quality of green coffee under the influence from different shade types and levels of fertilization and their correlation. Another purpose was to investigate the effect of the different agro ecosystems on the environmental conditions. Predominantly the production is less with cultivation under shade but it offers better conversion from cherries to green coffee and quality compared to cropping systems with full sun. Also the different shade tree species affect both production and environmental services. Coffee cultivation with regulated shade cover and adequate tree species combined with organic fertilization can achieve the same production level as cultivation at full sun with synthetic fertilizers. The combination of Simarouba glauca/Tabebuia rosea (SGTR) and the intensive organic fertilization results as the best treatment regarding the principal variables with an average production of $2674 \mathrm{~kg}$ oro ha ${ }^{-1}$ in the fifth crop 
especies arbóreas y nivel de sombra adecuada con un manejo orgánico intensivo pueden llegar a la misma producción que el cultivo a pleno sol con un tratamiento intensivo convencional. Adicional a esto, los sistemas con sombra mejoran la calidad y las condiciones ecológicas, también agregan valor por la madera, leña y frutos producidos y/o el pago por los servicios ambientales. El tratamiento SGTR brindó las mejores condiciones de hábitat y conservación de suelo, sin embargo, respecto a la fijación de carbono la combinación SSIL fue superior (24.41 SGTR vs 92.64 SSIL). Futuras investigaciones deberán evaluar más detalles sobre el efecto, uso y valor ecológico de especies de sombra y su influencia particular en la calidad en taza a diversas altitudes. Palabras clave: Simarouba glauca, Tabebuia rosea, correlación sombra y nutrición, rendimiento en uva y café oro, sistemas agroforestales, valoración ecológica, servicios ambientales, dióxido de carbono. (higher even than the full sun with a high level of synthetic fertilization). Furthermore, this kind of agroforestry system with regulated shade improves the quality and the conditions of the ecosystems and creates additional value from the shade trees (wood, firewood, fruits) and payments for environmental services. The shade SGTR offered the best conditions for the environment and the conservation of the soil, but with respect to the capture of carbon dioxide, with shade from Simanea saman + Inga laurina surpasses the other treatments containing $92.4 \mathrm{t} \mathrm{C}$ ha-1 against only 24.4 t C ha-1 in SGTR, or $4.7 \mathrm{t} \mathrm{C} \mathrm{ha}^{-1}$ in full sun coffee. Further investigations should focus on effect, benefit and ecological value of different shade tree species and their influence in the cup quality, also considering different altitudes. In addition greater awareness of the ecological issues needs to be developed from the farmer up to the consumer.
$\mathrm{E}$ 1 cultivo del café (Coffea arabica L.) es uno de los rubros más importantes en la economía $\mathrm{y}$ vida social de Nicaragua, representa cerca del $30 \%$ del PIB agrícola y $50 \%$ de las divisas provenientes de las exportaciones. Desde el punto de vista social emplea el $31.5 \%$ del total de la mano de obra agrícola.

El mercado de hoy, exige condiciones de protección ambiental y social en las fincas, ambos elementos le dan valor agregado al cultivo del café. En este sentido el establecimiento de especies arbóreas en el cafetal, lo convierte en un sistema diversificado, desde el punto de vista ambiental, por la diversidaddeárboles asociadosalaespeciecultivada(Muschler et al., 2006). La diferenciación del café por certificaciones como comercio justo, café orgánico o amigable con pájaros permite a los productores obtener un precio superior con respecto al mercado convencional (Bosselmann et al., 2009).

La producción de café bajo sombra es importante para crear un micro clima adecuado (humedad y temperatura), particularmente en áreas no óptimas para el cultivo, como las zonas bajas del Pacífico de Nicaragua (Bolaños et al., 2005).

El cambio climático es, sin duda, uno de los problemas ambientales más graves de nuestro tiempo y en las últimas dos décadas la humanidad ha intensificado su interés y su conciencia sobre el efecto invernadero provocado por las emisiones de $\mathrm{CO}_{2}$ (Pineda, et al., 2005). En general, los bosques son reconocidos como sistemas importantes para los servicios ambientales como: almacenamiento y captura de Carbono, producción de Oxigeno, belleza escénica, hábitat para diversas especies de animales, etc. Sin embargo, existen sistemas agroforestales productivos, el café entre ellos, que pueden llegar a tener similar valor ecológico.

Por la importancia del café como producto en el mercado mundial y en particular por la crisis de bajos precios en la década 1995-2005, se han realizado estudios para evaluar los efectos positivos del nivel de sombra en mejoras al rendimiento de café (tasa de conversión uva/pergamino/ oro) y la calidad. Pero, no hay trabajos enfocados a evaluar la influencia de las diferentes especies de árboles de sombra en la producción, rendimiento y calidad. Sobre esta base, el presente trabajo tiene como propósito mostrar, que diferentes calidades de sombra y diferentes niveles de insumos influyen en la producción, el rendimiento de grano oro y además determinar el valor ecológico de los sistemas en estudio.

\section{MATERIALES Y MÉTODOS}

Descripción del área de estudio. Se establecieron dos repeticiones de un ensayo con café en el año 2000 en áreas del Centro de Capacitación y Servicio Regional del Pacifico de Nicaragua (Jardín Botánico) de la Unión Nicaragüense de Cafetaleros (UNICAFE) ubicado en Masatepe a $453 \mathrm{msnm}$, $1400 \mathrm{~mm}$ de precipitación anual, temperaturas promedios de $24^{\circ} \mathrm{C}$ y $70-80 \%$ de humedad relativa. Los suelos son Humic Durustand con topografía plana, alto contenido de materia orgánica y acidez de $5-6$ de $\mathrm{pH}$ (Haggar et al., 2001). Una tercera repetición fue establecida en el año 2001 en áreas del Centro Experimental de Campo Azules (CECA) del INTA, localizado en la misma localidad.

Para el presente trabajo fueron seleccionados los tratamientos: combinación arbórea formada por Simarouba glauca (Acetuno) más Tabebuia rosea (Roble macuelizo) y Samanea saman (Genízaro) más Inga laurina (Guabilla) formando un gradiente de fijación de nitrógeno, contrastes de fenología, forma de dosel, usos económicos y ambientales. Estas combinaciones de sombra están subdividas en tipos y niveles de insumos: Convencional Intensivo (CI), Convencional Moderado (CM), Orgánico Intensivo (OI) y Orgánico Moderado (OM). Un tercer tipo de sombra evaluado fue "café a pleno sol" con uso de insumos convencionales en dos niveles: Convencional Intensivo y Moderado. La variedad usada en este ensayo fue Pacas, sembrado a $2 \times 1.25 \mathrm{~m}$; para una densidad poblacional de 4000 plantas ha $^{-1 .}$ 
Tabla 1. Diseño experimental del ensayo de sistemas agroforestales con café en Masatepe

\begin{tabular}{|c|c|c|c|c|c|}
\hline \multirow{2}{*}{$\begin{array}{l}\text { Parcela } \\
\text { principal }\end{array}$} & \multirow{2}{*}{$\begin{array}{l}\text { Café a } \\
\text { pleno sol } \\
\text { (PS) }\end{array}$} & \multicolumn{4}{|c|}{ Sistemas de café con diferentes sombras } \\
\hline & & $\begin{array}{l}\text { Simarouba glauca }+ \\
\text { Tabebuia rosea }(\text { SGTR) }\end{array}$ & $\begin{array}{l}\text { Tabebuia rosea }+ \\
\text { Samanea saman } \\
\text { (TRSS) }\end{array}$ & $\begin{array}{l}\text { Simarouba glauca } \\
+ \text { Inga laurina } \\
\text { (SGIL) }\end{array}$ & $\begin{array}{l}\text { Inga laurina }+ \\
\text { Samanea saman } \\
\text { (ILSS) }\end{array}$ \\
\hline $\begin{array}{c}\text { Sub } \\
\text { parcela }\end{array}$ & $\mathrm{CI}, \mathrm{CM}$ & $\mathrm{CI}, \mathrm{CM}, \mathrm{OI}, \mathrm{OM}$ & $\mathrm{CM}, \mathrm{OI}$ & CM, OI & CI, CM, OI, OM \\
\hline
\end{tabular}

\section{Variables medidas}

Producción y rendimiento. Se compararon tres ciclos de cosecha (2006 -2009), considerando que las tres repeticiones del ensayo no se establecieron en el mismo año, se formaron parejas de comparación: cosecha 4 (ciclo 05/06 de la repetición una y dos, ciclo 06/07 de repetición 3), cosecha 5 (ciclo 06/07 de la repetición uno y dos, ciclo 07/08 de repetición 3) y la cosecha $6 / 7$ (ciclo $08 / 09$ de las tres repeticiones).

Relación café uva/café oro. De cada tratamiento al momento de la cosecha se recolectó una muestra de dos "medios" de café uva, posteriormente se pesó y despulpó en una despulpadora manual. El café despulpado fue puesto a fermentar en una bolsa plástica por 16 horas. Una vez fermentado, se cuantificó el volumen y peso del café pergamino, posterior a ello se lavó y se pesó el café pergamino mojado; seguidamente el café fue secado en zarandas, hasta alcanzar el $12 \%$ de humedad (punto de trillo). Después de un mes de reposo, se trilló y peso el café oro y fueron estimadas las relaciones en café a café oro en kilogramos por hectárea.

Valoración ecológica. La valoración ecológica fue una investigación orientada a los diferentes tipos de sombra: SGTR, SSIL y PS. La metodología propuesta según Medina et al (2006) evaluó tres servicios ambientales a nivel del ensayo: biodiversidad, captura de carbono y conservación de suelo.

El servicio ambiental Biodiversidad incluye indicadores como calidad de hábitat, calidad de sombra, especies presentes, cantidad de epifitas, estratos vegetales y el uso de plaguicidas. Con respecto a la captura de Carbono, fueron medidos los árboles presentes en la parcela experimental según la especie, la altura y diámetro a la altura del pecho $(1.3 \mathrm{~m})$. Para café se midió la altura y el diámetro del tallo en 20 plantas por parcela. Fue determinada la biomasa por componente utilizando ecuaciones alométricas, por ejemplo:

\section{Para áboles:}

¿Biomasa de árbol $\left(\mathrm{kg} \mathrm{ha}^{-1}\right)=10^{\left(2.3408^{*} \log (D A P)-0.9578\right)}$

\section{Para cafetos:}

EBiomasa de planta $\left(\mathrm{kg} \mathrm{ha}^{-1}\right)=10 \quad(-1.15+\log (D B))+\left(0.54^{*} \log (h)\right)$
Donde: DAP $=$ Diámetro a la altura del pecho $(\mathrm{cm})$; $\mathrm{DB}=$ diámetro basal de planta de café a $15 \mathrm{~cm}$ del suelo $(\mathrm{cm}) ; \mathrm{h}=$ la altura total de una planta de café $(\mathrm{m})$.

Los resultados de los cálculos usando las formulas se multiplican por cinco en caso de los árboles y por la densidad de siembra en caso de los cafetos para tener las cantidades de la biomasa fijada por hectárea; esas cifras se multiplica por 0,48 (cafetos) y 0,5 (árboles) y se dividió entre 1000 para obtener el promedio de toneladas de carbono fijado por hectárea. Los indicadores se tomaron diferenciados por el uso de tierra en dos tipos de sombra y a pleno sol

En Conservación del suelo, se aplicaron el indicador de cobertura de suelo según la metodología punta de zapato (Staver, 2001). Las fuentes de agua se evaluaron a nivel de finca y su uso respecto al manejo de contaminación, evidencia de erosión o deslizamientos y obras de prevención de contaminación. Análisis de los datos. Con los datos obtenidos se realizaron análisis de varianza y separación de medias por DUNCAN. El proceso de ordenamiento, y análisis de datos se realizó utilizando hojas de cálculo y el programa SAS versión 9.2

\section{RESULTADOS Y DISCUSIÓN}

Producción grano oro $\left(\mathrm{kg} \mathrm{ha}^{-1}\right)$. No se detectaron diferencias estadísticas significativas para los tipos de sombra y niveles de insumos para el rendimiento de café $\mathrm{kg}$ oro ha-1. Sin embargo, el cultivo a pleno sol (PS) con insumo intensivo presenta mayor producción (Cosecha 4: 1855.05; Cosecha 5: 2377.3; Cosecha 6: 3682,46 kg oro ha-1 respectivamente). La mayor producción se atribuye a la mayor radiación, esto ha sido confirmado en estudios anteriores, esto no constituye una desventaja si se enfoca solo en el aumento de la producción.

Los resultados del presente trabajo indican que el café en sistemas agroforestales no reduce el rendimiento, sino que pueden ser similares a las de pleno sol y aún superarla, usando niveles adecuados de insumos. Aunque el rendimiento no fue estadísticamente diferente entre los tipos de sombra evaluados, se puede observar tendencias claras a favor del cultivo bajo sombra. La producción de café bajo sombra con la combinación de SGTR y uso intensivo de insumos orgánicos superó en rendimiento al tratamiento a pleno sol con uso intensivo de insumos convencionales (Tabla 2). 
La combinación con maderables SGTR obtuvo mayor rendimiento (Cosecha 4: 1445.14; Cosecha 5: 2105.39; Cosecha 6: $2822.35 \mathrm{~kg}$ ha-1, respectivamente) y a largo plazo un mejor rendimiento a diferencia del obtenido por la combinación de SSIL (Tabla 2); aunque estadísticamente no fueron diferentes $(\mathrm{P}>0.6126)$.
Al evaluar el factor insumo como única variable, se visualiza el potencial de un cultivo con insumos orgánicos. Se considera que el punto clave es la fertilización adecuada para mantener el ciclo de nutrientes, sin importar si la fuente es orgánica o convencional. En la producción del ciclo 08/09, se observan efectos estadísticos significativos entre los niveles de insumo ( $p>0.0427)$.

Tabla 2. Rendimiento grano oro $\left(\mathrm{kg}_{\mathrm{g}}\right.$ oro ha $\left.{ }^{-1}\right)$ según tipo de sombra y niveles de insumo

\begin{tabular}{|c|c|c|c|c|}
\hline \multirow[t]{2}{*}{ Niveles de insumos } & \multicolumn{2}{|c|}{ Cosecha 4} & \multirow[b]{2}{*}{ SSIL } & \multirow[b]{2}{*}{$\hat{\mathbf{Y}}$} \\
\hline & $\begin{array}{c}\text { Pleno } \\
\text { sol }\end{array}$ & SGTR & & \\
\hline Convencional Intensivo & 1855 & 1579 & 1319 & 1584 \\
\hline Convencional Moderado & 1633 & 1342 & 1468 & 1481 \\
\hline Orgánico Intensivo & --- & 1464 & 1256 & 1360 \\
\hline Orgánico Moderado & --- & 1445 & 1328 & 1387 \\
\hline$\hat{\bar{Y}}$ & 1744 & 1457 & 1343 & \\
\hline \multicolumn{5}{|c|}{ Cosecha 5} \\
\hline Convencional Intensivo & 2377 & 2260 & 1425 & 2021 \\
\hline Convencional Moderado & 2131 & 1917 & 1235 & 1761 \\
\hline Orgánico Intensivo & --- & 2674 & 1932 & 2303 \\
\hline Orgánico Moderado & --- & 1571 & 1238 & 1405 \\
\hline$\hat{\mathrm{Y}}$ & 2254 & 2105 & 1458 & \\
\hline \multicolumn{5}{|c|}{ Cosecha 6} \\
\hline Convencional Intensivo & 3682 & 2763 & 2296 & 2914 \\
\hline Convencional Moderado & 2701 & 2709 & 1857 & 2422 \\
\hline Orgánico Intensivo & --- & 3209 & 3166 & 3187 \\
\hline Orgánico Moderado & --- & 2608 & 2321 & 2464 \\
\hline$\hat{\mathrm{Y}}$ & 3192 & 2822 & 2410 & \\
\hline
\end{tabular}

SGTR: S. glauca + T. rosea

SSIL: S. saman + I. laurina

El rendimiento más alto fue registrada en el tratamiento orgánico intensivo (OI) $\left(3187,37 \mathrm{~kg} \mathrm{ha}^{-1}\right)$ y la más bajo en el convencional moderado $(\mathrm{CM})\left(2422,3 \mathrm{~kg} \mathrm{ha}^{-1}\right)$. La producción entre los tratamientos orgánicos y convencionales bajo el mismo nivel de insumo fue similar. Mientras, en la cosecha 5 , la producción entre los niveles de tratamiento de un mismo tipo de insumos tuvo diferencias de casi mil $\mathrm{kg}$ de café oro ha-1 (OI: $2303,19 \mathrm{~kg} \mathrm{ha}^{-1}$; OM: $1404,86 \mathrm{~kg} \mathrm{ha}^{-1}$ ) y fue significativo estadísticamente ( $\mathrm{p}>0.0468)$. En la cosecha 6, el rendimiento no fue influido por el tipo, ni por el nivel de insumo ( $>0.5449)$, reafirmando la tendencia de los ciclos previos.
Relación café uva/café oro (factor de conversión). La relación de café uva necesarias para producir una unidad de café oro, muestra que el tratamiento a pleno sol obtuvo la relación más alta que oscila entre 7.54:1 a 6.77:1, mientras, las dos combinaciones de sombra oscilan entre 6.42:1 a 6.84:1 (Tabla 3). Los tratamientos con sombra requieren menor cantidad de uva por unidad de café oro, lo cual es deseable. Con respecto a los niveles de insumos, las aplicaciones orgánicas (OM y OI) mostraron en la cosecha 4 un mejor rendimiento respecto a la aplicación de los insumos convencionales (CI y CM). En las cosechas 5 y 6 los rendimientos fueron similares en todos los tratamientos (Tabla 3). 
Tabla 3. Relación uva a oro $(\mathrm{kg})$ según tipo de sombra y nivel de insumos

\begin{tabular}{lcccc}
\hline Niveles de insumos & & & & \\
\hline Cosecha 4 & $\begin{array}{c}\text { Pleno } \\
\text { sol }\end{array}$ & SGTR & SSIL & $\hat{\mathbf{Y}}$ \\
& & & & \\
\hline Convencional Intensivo & 7.33 & 6.24 & 6.88 & 6.82 \\
Convencional Moderado & 7.77 & 6.48 & 6.59 & 6.94 \\
Orgánico Intensivo & --- & 6.63 & 6.62 & 6.62 \\
Orgánico Moderado & --- & 6.31 & 6.55 & 6.43 \\
$\hat{\hat{Y}}$ & 7.54 & 6.42 & 6.66 & \\
\hline
\end{tabular}

\section{Cosecha 5}

\begin{tabular}{lllll}
\hline Convencional Intensivo & 6.99 & 7.03 & 6.89 & 6.97 \\
Convencional Moderado & 7.29 & 7.34 & 6.75 & 7.12 \\
Orgánico Intensivo & --- & 7.02 & 6.76 & 6.89 \\
Orgánico Moderado & --- & 7.05 & 6.95 & 7.00 \\
\hline$\hat{Y}$ & & & & \\
\hline
\end{tabular}

\section{Cosecha 6}

\begin{tabular}{lllll}
\hline Convencional Intensivo & 6.96 & 6.43 & 6.72 & 6.70 \\
Convencional Moderado & 6.58 & 6.43 & 6.54 & 6.52 \\
Orgánico Intensivo & --- & 6.61 & 6.79 & 6.70 \\
Orgánico Moderado & --- & 6.55 & 6.40 & 6.48 \\
\hline$\hat{Y}$ & 6.77 & 6.51 & 6.61 &
\end{tabular}

SGTR: S. glauca + T. rosea

SSIL: S. saman + I. laurina

Correlación entre tipo de sombra y nivel de insumo. Con respecto a las interacciones de tipo de sombra e insumos, la combinación PS en interacción con CI y cosecha 08-09 se obtiene la mayor producción (2682.5 kg oro ha-1); mientras, la combinación SSIL en interacción con CM fue la mas baja con $1888.0 \mathrm{~kg}$ oro ha ${ }^{-1}$ (Figura 1). También se muestra que la combinación SGTR en interacción con OI supera en producción (3208.7 $\mathrm{kg}$ oro ha $\mathrm{h}^{-1}$ ) al CI con el mismo tipo de sombra (2763.4 kg oro ha ${ }^{-1)}$ y estuvo próximo a alcanzar el mismo nivel de producción a la combinación PS-CI (Figura 1).

La correlación mostrada a través de la variable conversión uva/oro, indica que en cafetales a plena exposición solar se requiere mayor cantidad de grano uva para producir una unidad de peso en oro (7.09:1 y 7.21:1). Este resultado es coherente en función de la producción oro alcanzado, mientras que las combinaciones de sombra obtienen una menor relación necesaria para producir una unidad de peso de grano oro (Tabla 4). En la correlación de sombra por insumo con respecto a la tasa de conversión (rendimiento) SSIL * OM fue la mejor combinación $(6,63: 1)$ y la más baja fue PS * CM $(7,21)$.

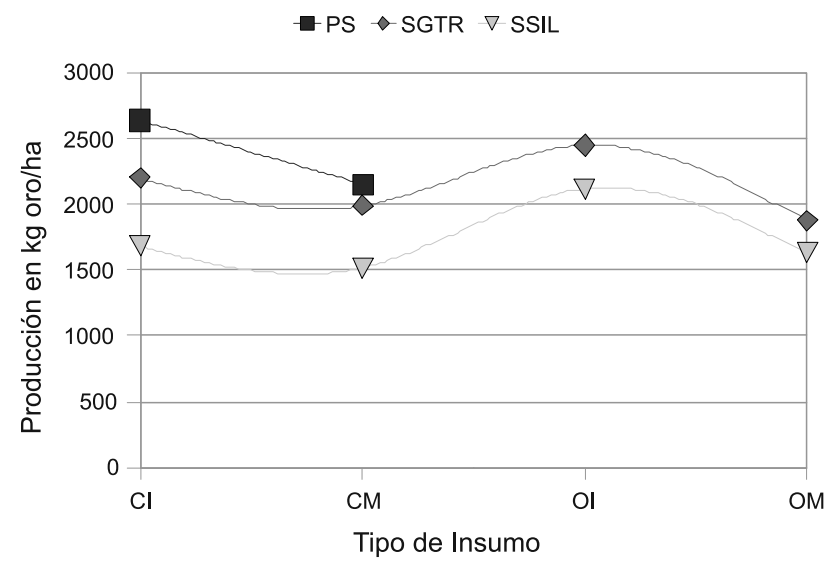

Figura 1. Correlación entre los tipos de sombra y niveles de insumo del promedio acumulado de producción.

Tabla 4. Correlación entre el tipo de sombra y niveles de insumo en la relación grano uva grano oro.

\begin{tabular}{lccc}
\hline Niveles de insumos & \multicolumn{3}{c}{ Tipo de sombra } \\
\hline & $\begin{array}{c}\text { Pleno } \\
\text { sol }\end{array}$ & SGTR & SSIL \\
& & & \\
\hline Convencional Intensivo & 7.09 & 6.57 & 6.83 \\
Convencional Moderado & 7.21 & 6.75 & 6.63 \\
Orgánico Intensivo & --- & 6.75 & 6.72 \\
Orgánico Moderado & --- & 6.64 & 6.63 \\
\hline
\end{tabular}

SGTR: $S$. glauca + T. rosea

SSIL: S. saman + I. laurina

Valoración de los servicios ambientales

Biodiversidad. La biodiversidad consiste en la gran variedad o pluralidad de organismos en la naturaleza en dichos ambientes (Soto-Pinto, 2006) y los cafetales agroforestales en comparación a otros cultivos, solo tienen beneficios para el ambiente menos la posible contaminación de los ríos por la pulpa de café. Con respecto a la calidad de hábitat y el uso de agroquímicos, en este estudio se presentó una diferencia muy clara entre las parcelas con árboles de sombra y el cultivo a pleno sol. También, se encuentran diferencias entre las combinaciones de especies arbóreas, SSTR fue superior 0.26 puntos con respecto a la combinación SSIL. Otros indicadores como el número de estratos en las combinaciones arbóreas tienen 3, lo cual está representado en los árboles presentes comparados con el café a pleno sol donde se denotan 2. Una situación importante en las combinaciones de sombra es que las especies presentes son nativas (Tabla 5). Según los resultados el nivel o la calidad del hábitat depende en gran parte de las especies arbóreas por su diferencia en la morfología, el dosel, su crecimiento y características como leguminosas o maderables. 
Captura de carbono. Los bosques son reconocidos como sistemas importantes en servicios ambientales como también en el almacenamiento y la captura de dióxido de carbono $\left(\mathrm{CO}_{2}\right)$ (Pineda-López et al., 2005). En las últimas dos décadas, la humanidad ha intensificado su interés sobre el efecto invernadero provocado por las emisiones de dióxido de carbono (Soto-Pinto et al., 2006).

El potencial de captura de carbono en áreas con sombra establecida es hasta 20 veces mas grande, que en el cultivo a pleno sol. Los resultados muestran en la combinación SSIL fue de 92.68 tn ha $^{-1}$ y solo 4.68 tn ha ${ }^{-1}$ en el cultivo a pleno sol. La combinación de SGTR fija mucho menos carbono con 24.41 tn $\mathrm{ha}^{-1}$, muestra la importancia del tipo de especie en cuanto a la captura de carbono; debido a las diferencias morfológicas, el crecimiento y la presencia de leguminosas o maderables. Otra variable importante es la densidad de árboles, tal es el caso de SSIL con la mayor densidad arbórea. El aporte de los cafetos a la captura de Carbono es mayor en café a plena exposición solar y el menor en la combinación arbórea SSIL (Tabla 5).

Una limitante del valor informativo del trabajo presente consiste en la falta de la medición de $\mathrm{CO}_{2}$ en hojarasca y en el suelo, que puede contribuir a un alto porcentaje del Carbono capturado.

No hay un valor oficial sobre el precio de una tonelada de $\mathrm{CO}_{2}$ capturada o no emitida, aunque algunas agencias multilaterales han establecido ciertos precios para los proyectos de reducción de emisiones financiados por ellas mismas (por ejemplo, hasta 2005, el Banco Mundial emplea un precio de US $\$ 5.00$ por tonelada de $\mathrm{CO}_{2}$ equivalente no emitida), el precio de la tonelada está sujeto a oferta y demanda de bonos de carbono en el mercado. Por ello, el Instituto Nacional de Ecología (2008), indica que los precios oscilan entre US $\$ 0.90$ hasta los 19.70 por tonelada de $\mathrm{CO}_{2}$.

Conservación de suelo. Las observaciones a nivel de finca presenta un terreno con una topografía plana, por consiguiente no hay presencia de erosión, deslizamientos y sedimentación; sobre esta base de información no hay prácticas conservacionistas, aparte de las coberturas vivas establecidas en los surcos del cafetal.

Los resultados obtenidos de acuerdo al muestreo punta de zapato presentaron en la cobertura de suelo muy significativo. En la combinación $S$. glauca mas $T$. rosea presentó la mayor cobertura viva con $81.5 \%$, el área a pleno sol con $60 \%$ y la combinación $S$. saman mas $I$. laurina con $26 \%$. Las áreas de los diferentes tratamientos se distinguieron por la distribución del tipo de cobertura, solo en café a pleno sol se encontraron hierbas de hoja ancha (26 $\%$ ) zacates (34\%). La sombra con SGTR presenta la mayoría de su cobertura por rastreras $(86.5 \%)$, al contrario con SSIL donde la cobertura en su mayor parte se compone de hojarasca (67\%) y no de cobertura viva; también el indicador suelo desnudo superó a los demás tratamientos $(28.5 \%)$.

Tabla 5. Valores obtenidos como potencial del servicio ambiental según tipos de sombra en café agroforestal.

\begin{tabular}{|c|c|c|c|c|}
\hline Servicio ambiental & Indicadores & $\begin{array}{c}\text { Pleno } \\
\text { sol }\end{array}$ & SGTR & SSIL \\
\hline \multirow[t]{6}{*}{ Biodiversidad } & Cobertura de sombra (\%) & 0.00 & 0.75 & 0.46 \\
\hline & Estratos de la vegetación & 2.00 & 3.00 & 3.00 \\
\hline & Especies nativas 0.0 & 2.00 & 2.00 & \\
\hline & Árboles y arbustos & 0.00 & 0.16 & 0.19 \\
\hline & Árboles con epifitas & 0.00 & 0.00 & 0.00 \\
\hline & Valor en Calidad de hábitat & 2.00 & 5.91 & 5.65 \\
\hline \multirow[t]{4}{*}{ Captura de carbono } & Árboles ha-1 & --- & 320.00 & 380.00 \\
\hline & Carbono en árboles (tn ha-1) & --- & 21.79 & 90.71 \\
\hline & Carbono en cafetos (tn ha-1) & 4.68 & 2.62 & 1.93 \\
\hline & Total de Carbono (tn ha-1) & 4.68 & 24.41 & 92.64 \\
\hline \multirow[t]{6}{*}{ Conservación de suelo } & Cobertura de suelo (\%) & 60.00 & 81.50 & 26.00 \\
\hline & Presencia de zacates & 34.00 & 0.00 & 0.00 \\
\hline & Hierbas hoja ancha & 26.00 & 0.00 & 0.00 \\
\hline & Hierbas rastreras & 0.00 & 86.50 & 54.50 \\
\hline & Hojarasca & 0.00 & 23.00 & 67.00 \\
\hline & Suelo desnudo & 0.00 & 5.00 & 28.50 \\
\hline \multicolumn{2}{|l|}{ Valor ecológico obtenido } & 6.00 & 8.15 & 2.6 \\
\hline
\end{tabular}




\section{CONCLUSIONES}

El rendimiento de café oro combinación Simaruba glauca mas Tabebu en la la producción supera la combinación de especies leguminosas bajo cualquier nivel de insumo. El rendimiento de café oro con niveles de fertilización orgánica adecuada puede ser igualmente productivo que el cultivo convencional con uso intensivo de insumos, inclusive sin sombra. Los agroecosistemas con árboles de café ofrecen un hábitat agradable para la fauna, logran conservar el suelo $\mathrm{y}$ tienen un potencial enorme para la fijación de Carbono.

\section{AGRADECIMIENTO}

Este trabajo ha sido posible gracias a la colaboración financiera del CATIE a través del proyecto Innovaciones con fondos del NORAD y a la contribución hecha por el personal de apoyo Elvin Navarrete y Leddis Navarrete.

\section{REFERENCIAS BIBLIOGRÁFICAS}

Bolaños, M; Moraga, P; Barrios, M; Haggar, J. 2005. Influence of shade types on quality an productivity of coffee in the Pacific zone of Nicaragua. UNICAFE / CATIE. Managua, Nicaragua.

Bosselmann S; A. Dons, K; Oberthur, T; Olsen S, C; Raebild, A; Usma, H. 2009. The influence of shade trees on coffee quality in small holder coffe agroforestry systems in Southern Colombia. In: Agriculture, Ecosystems an Environment, 129: 253 - 260.

FAO (Food and Agriculture Organization). 2004. Emergencia café: la FAO ayuda a los pequeños productores nicaraguenses: semillas y aperos de labranza para las familias más afectadas. Búsqueda de nuevos mercados. Disponible en http://www.fao.org/Newsroom/es/news/2004/35687/index.html

Haggar J. de Melo, E; Staver C. (2001) Sostenibilidad y sinergismo en sistemas agroforestales con café: estudio de interacciones entre plagas, fertilidad de suelo y árboles de sombra. Agroforestería en Las Américas, 8 (29) 49-51.

Medina F, B; Muñoz A, C; Haggar, J; Aguilar, R. 2006. Metodología para la evaluación de servicios ambientales. ANACAFE, Guatemala. $40 \mathrm{p}$.

Muschler, Reinhold G; Yépez, C; Rodríguez Camacho, A; Peters Grether, W; Pohlan H, AJ. 2006. Manejo y valoración de la biodiversidad de flora y fauna en cafetales. In: El Cafetal del Futuro: Realidades y Visiones (Eds., J. Pohlan, L. Soto, J. Barrera); pp. 333-360. Shaker Verlag.

Pineda-López, Ma; Ortiz-Ceballos, G; Sánchez-Velásquez, L. 2005. Los cafetales y su papel en la captura de carbono: un servicio ambiental aún no valorado en Veracruz. Madera y Bosques, 11(2): 3-14.

Soto-Pinto, L. 2006. Diversidad y otros servicios ambientales de los cafetales. ECOSUR, México. Disponible en: http// www.ecosur. mx/ecofronteras/ecofrontera/ecofront32/Diversidad.pdf.

Soto-Pinto, L; de Jong B, HJ.; Esquivel-Bazán, E; Sotero Quechulpa. 2006. Potencial ecológico y económico de captura de carbono en cafetales. In: El Cafetal del Futuro: Realidades y Visiones (Eds., J. Pohlan, L. Soto, J. Barrera); pp. $373-$ 380. Shaker Verlag.

Staver, Ch. 2001. Diagnóstico de las malas hiervas en los cafetales. 2da Edic. Edit. IMPASA. Managua, Nicaragua. 